\title{
How Many High Risk Korean Patients with Osteopenia Could Overlook Treatment Eligibility?
}

\author{
Dae-Hyun Yoon, Dong-Hyuk Choi, Hyun-Gyun Jung, Ju-Young Heo, Young-Jae Jang, Yong-Soo Choi \\ Department of Orthopedic Surgery, Kwangju Christian Hospital, Gwangju, Korea
}

\begin{abstract}
Study Design: Retrospective study.
Purpose: To determine the prevalence of high risk patient with osteopenia requiring pharmacologic treatment and investigate the difference of 10-year fracture probability whether bone mineral density (BMD) include or not in Korean FRAX model.

Overview of Literature: Many people with the fracture have osteopenia rather than osteoporosis, and BMD alone could be considered as a chance to prevent fracture.

Methods: Three hundred sixty-nine patients who was diagnosed as osteopenia were divided into two groups according to age (group 1 , under 65 years; group 2, over 65 years), and 10-year fracture probabilities were calculated by FRAX algorithm with and without femur neck T-score.

Results: The high risk patients of the fracture who had above 3\% of 10-year hip fracture probability were 15 cases in group 1 and 121 cases in group 2. In 193 patients of group 1, the mean 10-year fracture probability with BMD was significantly higher than the results without BMD (hip fracture: $p=0.04$, major osteoporotic fracture: $p=0.01$ ). Unlike the results of the group 1, the mean 10-year fracture probability without BMD was significantly higher than the results with BMD in 176 patients of group 2 (hip fracture: $p=0.01$, major osteoporotic fracture: $p=0.01$ ).

Conclusions: Total of 136 cases (36.8\%) as a high risk of the fracture with osteopenia could be overlooked treatment eligibility in Korean. The Korean FRAX model without BMD could be effective in predicting fracture risk especially in the individuals who were over 65 years.
\end{abstract}

Keywords: Osteopenia; Risk assessment; Bone mineral density; FRAX

\section{Introduction}

An important component of the fracture risk is not only low bone mass but also many clinical risk factors (CRF) that contribute to skeletal fragility $[1,2]$. Treatment intervention thresholds for prevention of osteoporotic fractures can be derived from criteria of the World Health Organization (WHO), minimum threshold with a lower score $(-2.5)$ compared to the standard T-score. However,
Wainwright et al. [3] reported the incident of hip fracture in 8,065 women with aged 65 year or older who were followed up to 5 years after bone mineral density (BMD) measurement. Among all participants, 17\% had osteoporosis. Of the 243 women with incident hip fracture, 54\% were not osteoporotic at start of follow-up. The women with osteopenia, however, have increased the risk of fracture $[4,5]$, and BMD alone could be overlooked treatment eligibility for fracture prevention.

Received Feb 15, 2014; Revised Mar 23, 2014; Accepted Mar 27, 2014

Corresponding author: Dong-Hyuk Choi

Department of Orthopedic Surgery, Kwangju Christian Hospital,

37 Yangnim-ro, Nam-gu, Gwangju 503-715, Korea

Tel: +82-62-650-5064, Fax: +82-62-650-5066, E-mail: hjmate@hanmail.net 
In 2008, the FRAX tool has been developed by WHO for estimating individualized 10-year probability of hip and major osteoporotic fracture [1]. It is based on individual patient models that integrate the risks associated with CRF (prior fragility fracture, parental hip fracture, smoking, systemic glucocorticoid use, excess alcohol intake, body mass index, rheumatoid arthritis, and other secondary causes of osteoporosis) as well as BMD at the femoral neck [6,7]. And the National Osteoporosis Foundation (NOF) recommended pharmacologic treatment to be considered in the osteopenia that had a 10-year hip fracture probability $\geq 3 \%$ or major fracture (humerus, forearm, hip or clinical vertebral fracture) probability $\geq 20 \%[8]$.

However, there is little disagreement for clinical application of the FRAX model. When BMD is included in the FRAX calculation, T-score of femoral neck must be used. Discordance between lumbar spine and femoral neck Tscore is common and BMD input in the FRAX model has a large effect on the result [9]. Another issue is treatment eligibility for a high risk Korean patient with the fracture on osteopenia. The Health Insurance Review Agency (HIRA) in Korea identifies treatment eligibility using a simplified method based on a minimum T-score $(-2.5)$ on a BMD examination or radiographic evidence of osteoporotic fractures [10]. The fixed T-score-dependent threshold for pharmacologic treatment is a simple and easy method for making national reimbursement decisions. And so, indeed the high risk Korean patient of the fracture with osteopenia could overlook pharmacologic treatment.

The purpose of this study was to determine the prevalence of high risk Korean patient with osteopenia who should take pharmacologic treatment according to Korean FRAX model and the NOF recommendation. In addition, we investigated the difference of the 10-year fracture probability whether BMD must be included or not in the Korean FRAX model.

\section{Materials and Methods}

The population for the study consisted of 369 individuals who checked a BMD in our hospital and were diagnosed as osteopenia from January 2008 to March 2011. Subjects who had already prescribed pharmacologic treatment for osteoporosis were excluded. The medical diagnoses were assessed through a combination of hospital dis- charge records (diagnoses and procedures coded using the Korean standard Classification of Diseases [KCD]-5). In this cross-sectional study, the survey was conducted telephone interviews for a questionnaire about the CRF of FRAX from January to March 2012. The risk factors included age, height, body weight, previous fragility fracture as an adult, family history of osteoporosis (parent with hip fracture), current smoking, glucocorticoid use, rheumatoid arthritis, secondary osteoporosis (examples given include type 1 diabetes, osteogenesis imperfecta in adults, untreated long-standing, hypothyroidism and hypogonadism or premature menopause) and more than 3 units of daily alcohol consumption. A prior fragility fracture was defined as a major osteoporotic fracture before a BMD examination. A diagnosis of rheumatoid arthritis was taken from physician office visits and/or hospitalizations with a relevant KCD-5 code in 3-year period prior to BMD examination. Prolonged corticosteroid use was defined as over 90 days dispended in the year prior to BMD examination at a mean prednisolone-equivalent dose of $7.5 \mathrm{mg}$ or greater per day.

Proximal femur (femoral neck, total hip, and trochanter) dual-energy X-ray absorptiometry (DXA) scans were performed and analyzed in accordance to manufacturer's recommendations. Weight and height were recorded at the time of DXA examination. Furthermore, this study was approved by Institutional Review Board at the Kwangju Christian Hospital.

The patients were divided into two groups depending on age (under 65 years as group 1, over 65 years as group 2). The NOF guide recommends BMD examination for all women age 65 and older. And younger women whose fracture risk is equal to or greater than that of a 65-year-old white woman who has no additional risk factors $[8,11]$. In Korea, The HIRA recommend BMD examination for women age 65 or older. Based on these backgrounds, we regarded age 65 years as the standard of categorization, 193 patients in group 1 and 176 patients in group 2. In all patients, 329 (89.1\%) patients were female, 177 patients in group 1 and 152 patients in group 2. In group 1, the mean age of 53.7 years and 71.6 years in group 2. A 10-year probability of hip and major fracture was calculated using the Korean FRAX model with and without femoral neck BMD for each group. In both groups, the 10-year fracture probabilities derived without $\mathrm{BMD}$ were compared to those that included BMD.

Descriptive statistics include means and standard de- 
viations (SD) for continuous variables. An independent $t$-test was used to compare the differences in age, height, weight, and femoral neck T-score between group 1 and group 2. Also, chi-square was used to compare differences in sex, previous fracture history, parents fracture history, smoking, alcohol, glucocorticoid, rheumatoid arthritis and secondary osteoporosis between group 1 and group 2. A paired $t$-test was used to compare difference of 10-year probabilities of fracture between FRAX model without T-score and with T-score. Statistical analyses were performed with SPSS for Windows ver. 12.0 (SPSS Inc., Chicago, IL, USA). The significance level was set at $p<0.05$.

\section{Results}

The high risk patient of the fracture with osteopenia who had above $3 \%$ of 10 -year hip fracture probability were observed in 15 cases in group 1 and 121 cases in group 2 when femoral neck T-score was not applied in FRAX calculation. On the other hand, it was decreased to 90 cases (group 1, 17: group 2, 73) when femoral neck T-score in FRAX calculation was added. Total of 136 cases $(36.8 \%)$ as a high risk of the fracture with osteopenia were overlooked treatment eligibility according to the NOF recommendation in Korea.

In 193 patients of group 1, the mean 10-year probability of hip fracture including femoral neck T-score was 1.39\% \pm $1.28 \%$ and the mean 10 -year probability of hip fracture without femoral neck T-score was $1.23 \% \pm 1.35 \%$. Also, the mean 10-year probability of major osteoporotic fracture including femoral neck T-score was $6.31 \% \pm 3.44 \%$, the mean 10-year probability of major osteoporotic fracture without femoral neck T-score was $5.73 \% \pm 3.49 \%$. The mean 10-year fracture probability with BMD was significantly higher than the results without BMD (hip fracture: $p=0.04$, major osteoporotic fracture: $p=0.01$ ) (Fig. 1). In 176 patients in group 2, the mean 10-year probability of hip fracture including femoral neck T-score was $3.47 \% \pm 4.43 \%$ and the mean 10 -year probability of hip fracture without femoral neck T-score was $5.92 \% \pm 6.69 \%$. Continuously, the mean 10 -year probability of major osteoporotic fracture including femoral neck T-score was $9.56 \% \pm 6.27 \%$, while the mean 10 -year probability of major osteoporotic fracture without femoral neck T-score was $12.21 \% \pm 8.39 \%$. Unlike the results of the group 1 , the mean 10-year fracture probability without BMD was significantly higher than the results with BMD (hip fracture: $p=0.01$, major osteoporotic fracture: $p=0.01$ ) (Fig. 2). Compared to the CRF of FRAX between two groups, there were higher CRF that contribute to the skeletal fragility in group 2 , such as height $(p=0.013)$, glucocorticoid use $(p=0.018)$, rheumatoid arthritis $(p=0.005)$, secondary osteoporosis $(p=0.001)$ (Table 1$)$.

\section{Discussion}

Bone mineral density alone is inadequate in evaluating the risk of osteoporotic fractures [1,2]. Therefore the NOF recommended that pharmacologic treatment must be considered in osteopenia individuals who had a 10 -year hip fracture probability $\geq 3 \%$ or major fracture (humerus, forearm, hip or clinical vertebral fracture) probability $\geq 20 \%$ [8]. According to the WHO criteria,

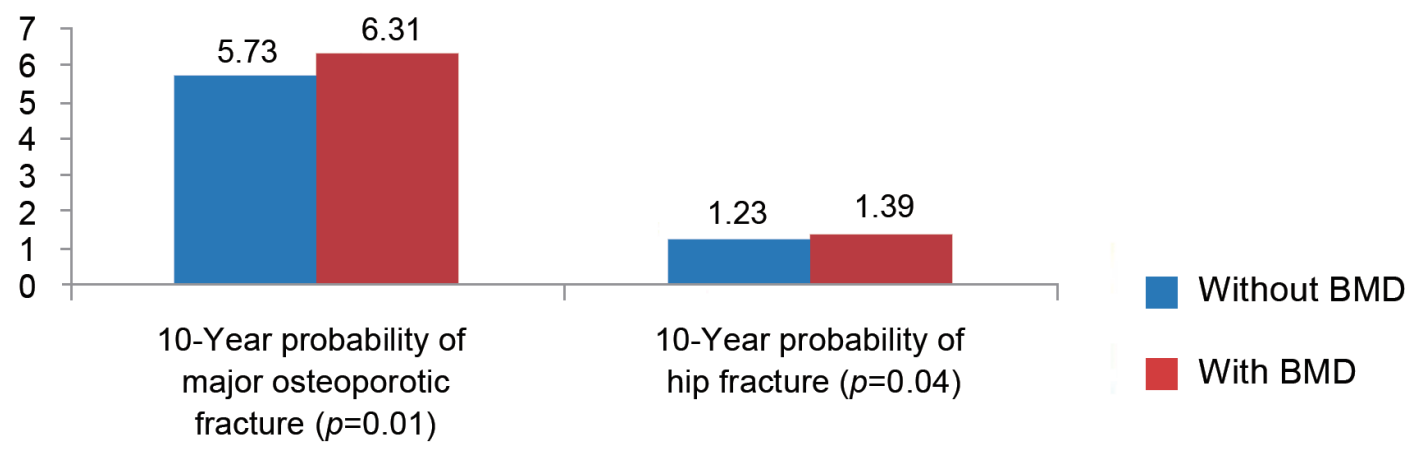

Fig. 1. Mean 10-year fracture probabilities of group 1, under 65-year-old. The mean 10-year fracture probability with bone mineral density (BMD) was significantly higher than the results without BMD (hip fracture: $p=0.04$, major osteoporotic fracture: $p=0.01)$. 
Table 1. The comparison of clinical risk factors of FRAX between two groups

\begin{tabular}{lccc} 
Characteristic & Group 1 (under 65-year-old) & Group 2 (over 65-year-old) & $p$-value \\
\hline Age $(\mathrm{yr})$ & 53.76 & 71.69 & 0.001 \\
Height $(\mathrm{cm})$ & 157.32 & 155.4 & 0.013 \\
Weight $(\mathrm{kg})$ & 56.58 & 57.11 & 0.502 \\
\hline Previous fracture history & 37 & 47 & 0.085 \\
\hline Parents fracture history & 12 & 13 & 0.655 \\
\hline Smoking & 7 & 11 & 0.243 \\
Alcohol $(\geq 3$ unit/day) & 9 & 37 & 0.747 \\
Glucocorticoid & 23 & 28 & 0.018 \\
\hline Rheumatoid arthritis & 13 & 99 & 0.005 \\
Secondary osteoporosis & 74 & -1.82 & 0.001 \\
\hline Femoral neck T-score & -1.65 & 0.001 & 0 \\
\hline
\end{tabular}

osteopenia is defined as a BMD that lies between 1.0 and 2.5 standard deviation below the average value for young healthy women $(1.0<\mathrm{T}$-score of $<-2.5 \mathrm{SD})$ [12].

In Koreans, according to our results, total of 136 cases $(36.8 \%)$ as a high risk of the fracture with osteopenia were overlooked pharmacologic treatment according the NOF recommendation. The HIRA has adopted a simple fixed T-score system to select a patient for pharmacological treatment, minimum threshold with a lower score $(-2.5)$ than the standard T-score. Our results according to the Korean FRAX model and the NOF recommendation demonstrated that the high-risk patients with osteopenia may be neglected in the current system that bases their judgment solely on T-score. Thus, CRF other than BMD should be considered to prevent the fracture.

The WHO developed the FRAX for estimating individ- ualized 10-year fracture probability [13]. FRAX identifies high-risk patients who respond to pharmaceutical intervention, irrespective of whether BMD was used in the calculation of fracture probability [14]. In other words, BMD is an option to calculate the fracture probability [15]. Although BMD is not required to calculate the fracture probability, FRAX results combining CRF with BMD have increased efficiency [16]. Other studies also have been noted that the fracture probability derived with $\mathrm{BMD}$ was higher than that without $\mathrm{BMD}$ or solely $\mathrm{BMD}$ $[15,17,18]$. Therefore we initially assumed that the fracture probability with BMD was higher than those without BMD. However, over 65-year-old group showed that the mean 10-year fracture probability without BMD was significantly higher than those with BMD. These results based on FRAX model suggest that fracture probability of

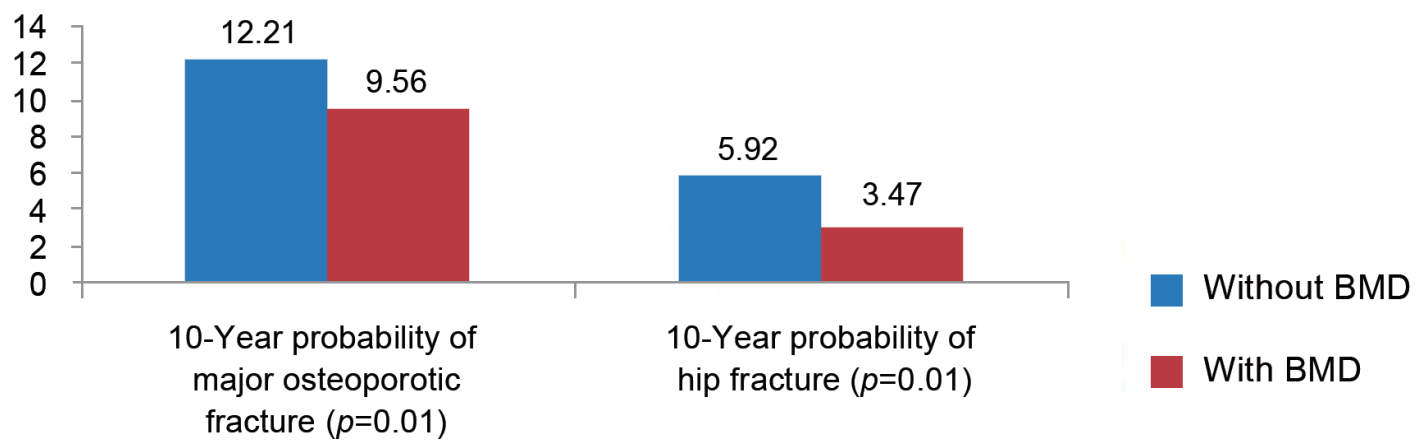

Fig. 2. Mean 10-year fracture probabilities of group 2, over 65-year-old. As opposed to the results of the group 1, the mean 10 year fracture probability without bone mineral density (BMD) was significantly higher than the results with BMD (hip fracture: $p=0.01$, major osteoporotic fracture: $p=0.01$ ). 
over 65-year-old group is associated with CRF that contribute to skeletal fragility as important component rather than BMD.

FRAX can be used without BMD when BMD is not available and to determine which patients qualify for BMD examination in some countries. BMD examination had many benefit for fracture risk reclassification of individuals who have an intermediate risk range of the FRAX [17]. Kanis et al. [14] assessed fracture prediction of FRAX with and without BMD and suggested that FRAX could be used without BMD in majority of the cases. They also insisted that BMD examination was reserved for individuals who were close to a probabilitybased intervention threshold. This study showed that Korean FRAX without BMD could be effective to predict fracture risk and assess treatment intervention in the osteopenia individuals who were specifically over 65 years. The FRAX can be also used as a screening tool to select individuals for subsequent BMD examination [19].

This study was limited by selection bias. Patients were recruited at one center and the nature of this study was retrospective collection and cross-sectional study. The sample size was small and this study couldn't suggest a guideline of intermediate risk for BMD examination according to Korean FRAX results. Further investigation is needed to clarify an intermediate risk of Korean FRAX for subsequent BMD examination using a large cohort study.

\section{Conclusions}

Total of 136 cases $(36.8 \%)$ as high risk patients of the fracture with the osteopenia were overlooked treatment eligibility in Korean. The Korean FRAX without BMD could be effective in predicting fracture risk and assessing treatment intervention in the osteopenia individuals who were especially over 65 years.

\section{Conflict of Interest}

No potential conflict of interest relevant to this article was reported.

\section{References}

1. Kanis JA, McCloskey EV, Johansson H, Oden A, Melton LJ 3rd, Khaltaev N. A reference standard for the description of osteoporosis. Bone 2008;42:46775 .

2. Siris ES, Miller PD, Barrett-Connor E, et al. Identification and fracture outcomes of undiagnosed low bone mineral density in postmenopausal women: results from the National Osteoporosis Risk Assessment. JAMA 2001;286:2815-22.

3. Wainwright SA, Marshall LM, Ensrud KE, et al. Hip fracture in women without osteoporosis. J Clin Endocrinol Metab 2005;90:2787-93.

4. Siris ES, Chen YT, Abbott TA, et al. Bone mineral density thresholds for pharmacological intervention to prevent fractures. Arch Intern Med 2004;164:110812.

5. Pasco JA, Seeman E, Henry MJ, Merriman EN, Nicholson GC, Kotowicz MA. The population burden of fractures originates in women with osteopenia, not osteoporosis. Osteoporos Int 2006;17:1404-9.

6. Kanis JA, Borgstrom F, De Laet C, et al. Assessment of fracture risk. Osteoporos Int 2005;16:581-9.

7. Kanis JA, Johnell O, Oden A, Johansson H, McCloskey E. FRAX and the assessment of fracture probability in men and women from the UK. Osteoporos Int 2008;19:385-97.

8. National Osteoporosis Foundation. Clinician's guide to prevention and treatment of osteoporosis. Washington, DC: National Osteoporosis Foundation; 2013.

9. So GY, Park KH, Yoon DH, Ryu JH, Choi YS. Feasibility of FRAX for prediction of osteoporotic vertebral fractures in Korea. Asian Spine J 2012;6:22-8.

10. Korean Health Insurance Review Agency. General guideline of pharmacologic intervention for osteoporosis [Internet]. Seoul (KR): Health Insurance Review Agency; c2010 [cited 2010 May 20]. Available from: http://www.hira.or.kr.

11. Nelson HD, Haney EM, Chou R, Dana T, Fu R, Bougatsos C. Screening for Osteoporosis: Systematic Review to Update the 2002 U.S. Preventive Services Task Force Recommendation. Evidence Synthesis No. 77. AHRQ Publication No. 10-05145-EF-1. Rockville, Maryland: Agency for Healthcare Research and Quality; 2010.

12. Kanis JA, Melton LJ 3rd, Christiansen C, Johnston CC, Khaltaev N. The diagnosis of osteoporosis. J Bone Miner Res 1994;9:1137-41.

13. World Health Organization. WHO scientific group on the assessment of osteoporosis at primary health 
care level [Internet]. Geneva: WHO Press; 2004 [cited 2014 Nov 3]. Available from: http://www.who.int/ chp/topics/Osteoporosis.pdf.

14. Kanis JA, McCloskey E, Johansson H, Oden A, Leslie WD. $\operatorname{FRAX}((\mathrm{R}))$ with and without bone mineral density. Calcif Tissue Int 2012;90:1-13.

15. Kanis JA, Oden A, Johnell O, et al. The use of clinical risk factors enhances the performance of BMD in the prediction of hip and osteoporotic fractures in men and women. Osteoporos Int 2007;18:1033-46.

16. De Laet C, Oden A, Johansson H, Johnell O, Jonsson $B$, Kanis JA. The impact of the use of multiple risk indicators for fracture on case-finding strategies: a mathematical approach. Osteoporos Int 2005;16:3138.

17. Leslie WD, Morin S, Lix LM, et al. Fracture risk assessment without bone density measurement in routine clinical practice. Osteoporos Int 2012;23:75-85.

18. Leslie WD, Lix LM, Johansson H, et al. Independent clinical validation of a Canadian FRAX tool: fracture prediction and model calibration. J Bone Miner Res 2010;25:2350-8.

19. Kanis JA, McCloskey EV, Johansson H, et al. Case finding for the management of osteoporosis with FRAX--assessment and intervention thresholds for the UK. Osteoporos Int 2008;19:1395-408. 